\title{
Cardiac arrest caused by contralateral tension pneumothorax during one-lung ventilation - A case report -
}

Received May 15, 2019

Revised 1st, May 29, 2019

2nd, May 30, 2019

Accepted May 31, 2019

\section{Soo Kyung Lee, Kwon Hui Seo, You Jung Kim, Eun Ji Youn, Jun Suck Lee, Jieun Park, and Hyun Soo Moon}

Department of Anesthesiology and Pain Medicine, College of Medicine, Hallym University, Chuncheon, Korea

\begin{abstract}
Background: Tension pneumothorax on the contralateral lung during one-lung ventilation (OLV) can be life-threatening if not rapidly diagnosed and managed. However, diagnosis is often delayed because the classic signs of tension pneumothorax are similar to clinical manifestations commonly observed during OLV.
\end{abstract}

Case: We report a case of contralateral tension pneumothorax in a patient undergoing right upper lobectomy during OLV. The patient suffered from sudden cardiac arrest and was assisted by extra-corporeal membrane oxygenation.

Conclusions: Contralateral pneumothorax during OLV is rare but can occur at any time. Therefore, anesthesiologists should consider this critical complication.

Keywords: Heart arrest; One-lung ventilation; Pneumothorax; Thoracic surgery.
Contralateral tension pneumothorax during one-lung ventilation (OLV) is a rare event. As this condition can rapidly lead to a life-threatening situation, early diagnosis and immediate treatment are important. However, signs of tension pneumothorax such as hypoxemia, increased airway pressure or hypotension [1], occur frequently during OLV, often leading to delayed diagnosis. In the present work, we report a case of a patient with sudden cardiovascular collapse due to tension pneumothorax on the contralateral lung during OLV for lobectomy. The patient was kept on extra-corporeal membrane oxygenation (ECMO) support until the cause of respiratory and hemodynamic failure was identified and recovered. We obtained informed consent from patient.

\section{CASE REPORT}

A 75-year-old male weighing $50 \mathrm{~kg}$ and having a height of $165 \mathrm{~cm}$ was scheduled to undergo right upper lobectomy for the treatment of non-small cell lung cancer. His significant medical history was chronic obstructive pulmonary disease (COPD) and was a 55-pack-year smoker. The preoperative pulmonary function test showed a moderate obstructive pattern with a forced expiratory volume of $1.64 \mathrm{~L}$ (61\% of predicted) in one second. The chest computerized tomography revealed subsegmental atelectasis in bilateral lower lobes and right middle lobe, emphysema with bullae in bilateral lungs (Fig. 1), and scanty right pleural effusion. An electrocardiogram (ECG) performed before the surgery showed right bundle branch block, and transthoracic echocardiography revealed normal left ventricle (LV) global systolic function (ejection fraction $[\mathrm{EF}]=58 \%$ ) and abnormal relaxation of $\mathrm{LV}$ filling pattern.

The patient received $0.2 \mathrm{mg}$ of glycopyrrolate intramuscularly as a premedication. On arrival in the operating room, intraoperative monitoring was performed including ECG, noninvasive blood pressure, pulse oximetry, bispectral index,

This is an Open Access article distributed under the terms of the Creative Commons Attribution Non-Commercial License (http://creativecommons.org/licenses/by-nc/4.0) which permits unrestricted non-commercial use, distribution, and reproduction in any medium, provided the original work is properly cited. 


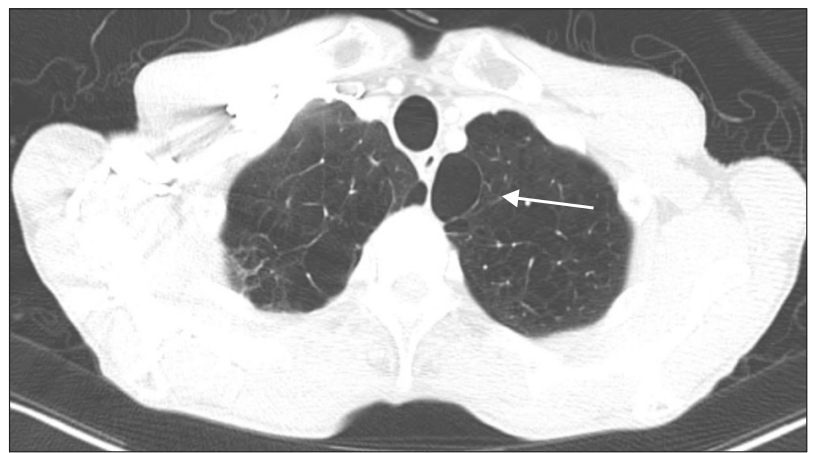

Fig. 1. Chest computed tomography shows emphysema with bullae in the bilateral lung. Arrow indicates the large bulla on the left lung.

and capnography. The immediate preoperative vital signs of the patient were as follows: systolic/diastolic blood pressure of 112/74 mmHg, a heart rate of 90 beats/min, and Oxygen saturation $\left(\mathrm{SpO}_{2}\right)$ of $96 \%$. After preoxygenation with $100 \%$ oxygen, general anesthesia was induced with intravenous 70 $\mathrm{mg}$ of propofol, $20 \mathrm{mg}$ of lidocaine, and continuous infusion of remifentanil. After confirming adequate mask ventilation, $60 \mathrm{mg}$ of succinylcholine was given to facilitate passage of a double-lumen endotracheal tube (DLT), after which neuromuscular blockade was maintained with intermittent boluses of intravenous rocuronium. The trachea was intubated with a 37 Fr. left-side DLT (Mallinckrodt ${ }^{\mathrm{TM}}$, Covidien Inc., Ireland) under direct laryngoscopy. The DLT position was confirmed by chest auscultation and fiberoptic bronchoscopy. A left radial artery catheter and a central venous catheter were placed via the right internal jugular vein. Arterial blood gas analysis (ABGA) during two-lung ventilation $\left(\mathrm{FiO}_{2}\right.$ 0.6/end-tidal $\mathrm{CO}_{2}$ concentration $\left[\mathrm{EtCO}_{2}\right], 34 \mathrm{mmHg}$ ) revealed $\mathrm{pH}$ 7.369, $\mathrm{PaCO}_{2}$ $40.6 \mathrm{mmHg}, \mathrm{PaO}_{2} 256.3 \mathrm{mmHg}$, and $\mathrm{SaO}_{2} 99.8 \%$. The patient was placed in the left lateral decubitus position for the planned video-assisted thoracoscopic surgery (VATS), and DLT positioning was again confirmed with fiberoptic bronchoscopy.

General anesthesia was maintained with balanced anesthesia using oxygen-air-sevoflurane (1-2 vol\%) and continuous infusion of remifentanil. After endotracheal intubation, $40 \mathrm{mg}$ of rocuronium was administered and an additional dose of $10 \mathrm{mg}$ was administered twice until the event described below occurred. Mechanical ventilation during OLV was initiated using pressure-controlled ventilation-volume guaranteed mode with a tidal volume of $300 \mathrm{ml}$ and a respiratory rate of 14 breaths/min. After about $30 \mathrm{~min}$ of procedure,
VATS was converted to thoracotomy due to surgical site adhesion. Peak airway pressure (PAP) was $14 \mathrm{cmH}_{2} \mathrm{O}$ during two-lung ventilation. After OLV, the PAP was $25 \mathrm{cmH}_{2} \mathrm{O}$ initially and gradually increased to $35 \mathrm{cmH}_{2} \mathrm{O}$ within $10 \mathrm{~min}$. $\mathrm{SpO}_{2}$ gradually decreased from $97 \%$ to $80 \%$ during the next $5 \mathrm{~min}$. As a rescue measure, two-lung ventilation was performed for several minutes and $\mathrm{SpO}_{2}$ increased to $96 \%$. After reapplication of the $\mathrm{OLV}, \mathrm{SpO}_{2}$ gradually decreased from $98 \%$ to $70 \%$. Inspired oxygen was increased from $60 \%$ to $100 \%$, and two lung ventilation was reinstituted. Oxygen saturation increased to $95 \%$ for approximately $5 \mathrm{~min}$. Systolic blood pressure decreased to $70 \mathrm{mmHg}$; subsequently, $100 \mu \mathrm{g}$ of phenylephrine was administered twice and infused continuously. However, blood pressure was maintained as $85 / 60$ mmHg. The ABGA $\left(\mathrm{FiO}_{2} 1.0, \mathrm{EtCO}_{2} 23 \mathrm{mmHg}\right)$ was checked and the outcomes were $\mathrm{pH} 7.223, \mathrm{PaCO}_{2} 60.9 \mathrm{mmHg}, \mathrm{PaO}_{2}$ $61.9 \mathrm{mmHg}$, and $\mathrm{SaO}_{2} 85.6 \%$. Dopamine infusion $(10 \mu \mathrm{g} / \mathrm{kg} /$ min) was started and systolic blood pressure was maintained above $100 \mathrm{mmHg}$. With intermittent suction of the DLT, oxygen insufflation into the non-dependent lung and dopamine administration, $\mathrm{SpO}_{2}$ was maintained between $85-90 \%$ during OLV. Subsequently, the PAP was noted to be about $35 \mathrm{cmH}_{2} \mathrm{O}$ (maximum $37 \mathrm{cmH}_{2} \mathrm{O}$ ). We did not apply positive end-expiratory pressure to the dependent lung because of the high PAP. Nearly $2 \mathrm{~h}$ after the operation, systolic blood pressure suddenly dropped below $80 \mathrm{mmHg}$ and fell below 50 mmHg within $1 \mathrm{~min}, \mathrm{SpO}_{2}$ decreased from $90 \%$ to $70 \%$, and $\mathrm{EtCO}_{2}$ decreased to $17-18 \mathrm{mmHg}$. Almost immediately, the arterial waveform disappeared and asystole was revealed on ECG. Two-lung ventilation was reinstituted and the surgical site was temporarily closed using dressing films. The patient was placed in the supine position and resuscitated with atropine and epinephrine, and the surgeon started closed cardiac compression. At the same time, we asked for help in applying ECMO. About 17 min later, recovery of spontaneous circulation achieved. Nearly at the same time, veno-arterial (VA) ECMO was also started via the right common femoral artery and common femoral vein. On auscultation, lung sound of the left lung field was decreased markedly. Tension pneumothorax of the dependent left lung was suspected. Portable chest X-ray revealed tension pneumothorax of the left lung (Fig. 2) and a chest tube was inserted immediately. Followup chest X-ray showed re-expansion of both the lungs (Fig. 3 ). The patient was returned to the left lateral decubitus posi- 


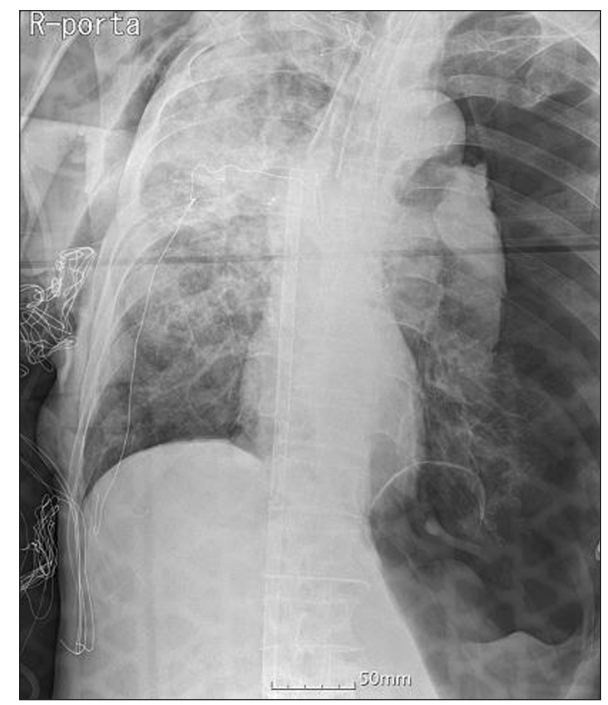

Fig. 2. Chest anteroposterior shows marked pneumothorax in the left lung.

tion, and the right upper lobectomy was performed after the right lung was selectively collapsed. At this time, the left lung was ventilated with a tidal volume of $250 \mathrm{ml}$ and a respiratory rate of 13 breaths/min, and PAP was about $35 \mathrm{cmH}_{2} \mathrm{O}$. After completion of the surgical procedure, the DLT was replaced with a single lumen endotracheal tube of $7.5 \mathrm{~mm}$ internal diameter, and the patient was hemodynamically stable. He was transferred to the intensive care unit while maintaining VAECMO.

Immediate postoperative echocardiography revealed moderate LV global and severe right ventricle (RV) free wall motion abnormality and decreased LV systolic function (EF $=40 \%)$. On the 10th day, transthoracic echocardiography showed normal LV global wall motion but still severe RV free wall motion abnormality was apparant. On the 14th day, echocardiography revealed normal RV free wall motion. Finally, ECMO was weaned off and the patient's trachea was extubated.

\section{DISCUSSION}

Tension pneumothorax is an uncommon condition that can be fatal if left untreated [2]. Intraoperative tension pneumothorax can be extremely dangerous, especially during OLV and is a rare complication. It can cause sudden death or serious long-term organ damage. There exist a few case reports in the literature on contralateral tension pneumothorax

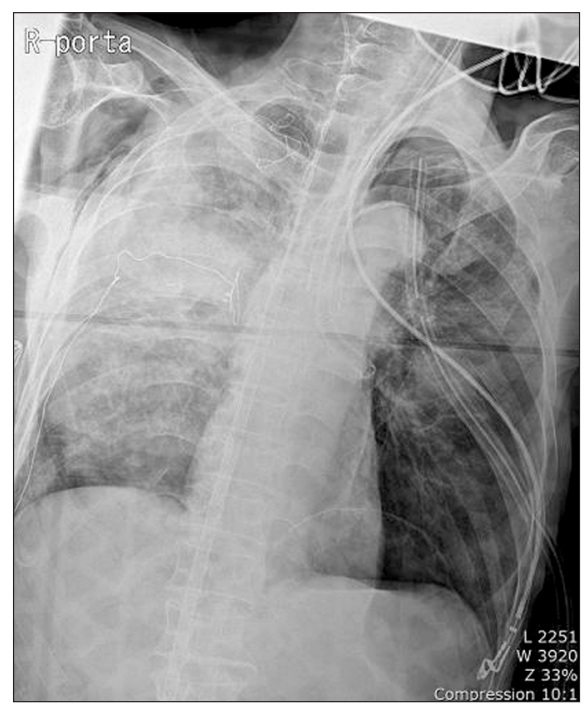

Fig. 3. Chest anteroposterior immediately after insertion of the chest tube shows re-expansion of the left lung.

during OLV in recent decades. Hoechter et al. [3] reported that there were only 9 cases published in the literature so far in 2018. However, the authors reported pneumothoraces in 4 of 2,494 patients who underwent OLV for 5.5 years and mentioned the possibility of underreporting.

Clinical manifestations of tension pneumothorax include an immediate and progressive decrease in $\mathrm{SpO}_{2}$, immediate reduction in cardiac output, increase in airway pressure, ventilatory disturbances of the ipsilateral lung, venous distension, and loss of breathing sounds on the affected side in patients undergoing mechanical ventilation [1,4-8]. These clinical findings are caused by shifting or compression of mediastinal structures and the lung parenchyma, or the presence of air in anatomic spaces. Decreased cardiac output is probably due to a combination of hypoxemia, reduced venous return, and compression of great the vessel, ventricle, and opposite lung [5]. If the pneumothorax is not recognized and treated urgently, the condition might lead to sudden cardiac arrest [1]. In the present case, cardiac arrest occurred within 3 minutes of the drop in blood pressure. These signs may not be apparent if the opposite chest is open during a surgical procedure, because mediastinal shift may not compress these structures to the extent when the chest is closed [5]. Finlayson et al. [4] reported a case of a patient with contralateral tension pneumothorax during thoracotomy without typical signs of marked hypoxemia and hemodynamic instability. The patient may show clinical manifestations only after the chest is 
closed [5]. In some cases, patients can show increased airway pressure and decreased oxygen saturation with only transient hypotension [9]. Therefore, even in the absence of typical signs such as hypoxemia and hypotension, the possibility of tension pneumothorax should not be overlooked in any patient who develops high peak inspiratory pressures during OLV with an open chest [4]. However, in the present case, the left tension pneumothorax prevented adequate venous return, even when the right chest was opened, resulting in complete cardiovascular collapse, as also reported by other authors $[6,7,10,11]$. Finlayson et al. [4] reported that in half of the cases, the cardiovascular collapse occurred before decompression of the pneumothorax.

Diagnosis is often delayed because the classic signs of tension pneumothorax are similar to the common clinical manifestations during OLV. Other causes such as DLT malposition, bronchial occlusion by secretions or blood, and dynamic hyperinflation of the lung are usually considered first [4]. In addition, the lateral decubitus position and surgical drapes make access to the dependent hemithorax for physical examination difficult. Checking the collapsed bronchus with a fiberoptic bronchoscopy can also help with diagnosis $[9,12]$ before confirming tension pneumothorax with chest radiography. In the present case, two-lung ventilation was resumed because of the possibility of tension pneumothorax, but cardiac arrest occurred quickly. Consequently, we started cardiopulmonary resuscitation after placing the patient in a supine position and asked the ECMO team for help. There exist a report which states that mediastinal thoracentesis was carried out without a definite diagnosis in nearly fatal case, as diagnostic methods took time and there was no time to delay the treatment [13]. However, in most of the cases, a diagnostic method such as chest X-ray or ultrasound is used to identify pneumothorax before attempting decompression.

In the present case, barotraumas is hypothesized as the possible cause of tension pneumothorax and it may occur as a result of air trapping within the dependent lung, leading to hyperinflation and rupture of bulla [12]. In previous case reports on tension pneumothorax during OLV, many cases were associated with barotraumas [3-7,10-14]. Some of them were related to inappropriate DLT size $[7,10,12]$, and some were related to rupture of bulla [12,13]. Sometimes patients undergoing lung surgery have pre-existing pulmonary conditions such as emphysema or bulla. In the diseased lungs, reduction in elasticity and compliance occur and the high ventilation pressures can affect the vulnerable lungs and lead to pneumothorax [3]. In patients with chronic obstructive lung disease and pulmonary hyperinflation, intrinsic positive end-expiratory pressure is common during OLV, which can lead to an excessive increase in airway pressure and decrease in tidal volume during OLV [15]. In this case, the patient had COPD accompanied by emphysema with bullae in bilateral lungs. Therefore, our patient had a risk of pneumothorax. Use of recruitment maneuvering techniques at high pressures can cause pneumothorax with barotraumas $[4,5]$.

In conclusion, contralateral pneumothorax during OLV is rare but can occur at any time. It may be associated with sudden death or serious long-term complications. Hoechter et al. [3] noted that only four out of the nine previously reported cases led to a correct diagnosis with clinical suspicion. Therefore, anesthesiologists should consider, be alert, and cope with this rare but critical complication.

\section{CONFLICTS OF INTEREST}

No potential conflict of interest relevant to this article was reported.

\section{ORCID}

Kwon Hui Seo, https://orcid.org/0000-0003-4397-9207

You Jung Kim, https://orcid.org/0000-0002-5652-1660

Eun Ji Youn, https://orcid.org/0000-0003-2842-9987

Jun Suck Lee, https://orcid.org/0000-0002-9752-9861

Jieun Park, https://orcid.org/0000-0001-6754-8802

Hyun Soo Moon, https://orcid.org/0000-0001-7623-4442

\section{REFERENCES}

1. Leigh-Smith S, Harris T. Tension pneumothorax--time for a rethink? Emerg Med J 2005; 22: 8-16.

2. Rojas R, Wasserberger J, Balasubramaniam S. Unsuspected tension pneumothorax as a hidden cause of unsuccessful resuscitation. Ann Emerg Med 1983; 12: 411-2.

3. Hoechter DJ, Speck E, Siegl D, Laven H, Zwissler B, Kammerer T. Tension pneumothorax during one-lung ventilation - an underestimated complication? J Cardiothorac Vasc Anesth 2018; 32: 1398-402.

4. Finlayson GN, Chiang AB, Brodsky JB, Cannon WB. Intraopera- 
tive contralateral tension pneumothorax during pneumonectomy. Anesth Analg 2008; 106: 58-60.

5. Gabbott DA, Carter JA. Contralateral tension pneumothorax during thoracotomy for lung resection. Anaesthesia 1990; 45: 229-31.

6. Fossard JP, Samet A, Meistelman C, Longrois D. Life-threatening pneumothorax of the ventilated lung during thoracoscopic pleurectomy. Can J Anaesth 2001; 48: 493-6.

7. Sivalingam P, Tio R. Tension pneumothorax, pneumomediastinum, pneumoperitoneum, and subcutaneous emphysema in a 15-year-old Chinese girl after a double-lumen tube intubation and one-lung ventilation. J Cardiothorac Vasc Anesth 1999; 13: 312-5.

8. Zollinger A, Zaugg M, Weder W, Russi EW, Blumenthal S, Zalunardo MP, et al. Video-assisted thoracoscopic volume reduction surgery in patients with diffuse pulmonary emphysema: gas exchange and anesthesiological management. Anesth Analg 1997; 84: 845-51.

9. Kim JY, Kim JH, Lim CH, Lee HW, Lim HJ, Yoon SM, et al. Tension pneumothorax during one-lung ventilation in patient with bronchiectasis: a case report. Korean J Anesthesiol 2005; 49: 11923.
10. Weng W, DeCrosta DJ, Zhang H. Tension pneumothorax during one-lung ventilation: a case report. J Clin Anesth 2002; 14: 52931.

11. Ryu SM, Na HS, Bae JH, Hong JM, Lim SW. Cardiac arrest occurred by tension pneumothorax during right thoracotomy: a case report. Korean J Anesthesiol 2006; 51: 756-9.

12. Malik S, Shapiro WA, Jablons D, Katz JA. Contralateral tension pneumothorax during one-lung ventilation for lobectomy: diagnosis aided by fiberoptic bronchoscopy. Anesth Analg 2002; 95 : $570-2$.

13. Kenta O, Shoko A, Takeshi I, Satoshi H, Yuji F, Yasushi S, et al. Dependent lung tension pneumothorax during 1-lung ventilation: treatment by transmediastinal thoracentesis. A A Case Rep 2015; 5: 61-3.

14. Arai H, Tajiri M, Ebuchi K, Ando K, Okudela K, Gamo M, et al. Contralateral tension pneumothorax during video-assisted thorascoscopic surgery for lung cancer: a case report. Clin Respir J 2018; 12: 298-301.

15. Bardoczky GI, Yernault JC, Engelman EE, Velghe CE, Cappello M, Hollander AA. Intrinsic positive end-expiratory pressure during one-lung ventilation for thoracic surgery. The influence of preoperative pulmonary function. Chest 1996; 110: 180-4. 